\title{
KINEMATIC SINGULARITY ANALYSIS AND SIMULATION FOR 7DOF ANTHROPOMORPHIC MANIPULATOR
}

\author{
Leigang ZHANG, Shuai GUO, Yingchao HUANG, Xuesheng XIONG \\ Shanghai Key Laboratory of Intelligent Manufacturing and Robotics, Shanghai University, Shanghai, China \\ Email: lgzhang@shu.edu.cn
}

\begin{abstract}
The determination and analysis of the singular or velocity-degenerate configurations of the KUKA LBR iiwa 7 R800 robot is presented. The KUKA LBR iiwa 7 R800 robot is a 7 -joint serial lightweight manipulator. Firstly,we constructed the kinematics model for iiwa robot and the DH parameters was derived. Secondly, theJacobeanmatrix was computed based on screw theory and the concept of screw reciprocity was used to find the singular (velocity-degenerate) configurations. Finally, the dexterity of end reference point of iiwa robot was analysed based on manipulability index and the minimum singular value index by means of the Robotics Toolbox.The analysis results show that there are four sigular conditions of the KUKA LBR iiwa 7 R800 robot within its workspace, the simulation results verified the correctness of the analysis in this article, and it lays a fonundation for subsequent singularity avoidance research.
\end{abstract}

Keywords: Jacobian; Dexterity; Singularities; Reciprocal Screws; Robotics Toolbox.

\section{Introduction}

Generally, a redundant manipulator which has more than necessary degrees of freedom (DOF) is more dexterous and versatile than a non redundant manipulator. However, both have the singularity problem.

The kinematic characteristics of robot including the singularity, workspace, dexterity, stability and so on, the singularity which is the inherent characteristic of robots is one of the most complex problem and can't be avoid. A singular or velocitydegenerate configuration is a configuration in which a robotic manipulator has lost at least one DOF of motion capability. At a singular configuration, the manipulator unable to excute an arbitrary instantaneous motion. In this case, if there still a velocity component of the end-effector in singular direction, the joint velocities of manipulator will become unacceptable large, and end-effector will deviate from its expected trajectory. Moreover, it is possible that a robot will be out of control $[1,2]$.Therefore, in order to ensure the stability and reliability of robot, the determination of singular configuration is critical to understanding a manipulator's kinematics and can be important in the implementation of a controller for the manipulator.

Robot singularity analysis, as the basis of singularity avoidance, is used to determine the singularity conditions. For non redundant robots, the determinant value of Jacobean matrix can directly determine robot singularity conditions. However, for redundant robot, because its Jacobean matrix is not square, the singularity analysis becomes much more complex. The singularity analysis method of robot mainly includes algebraic theory, screw theory and line geography theory. In recent years, much effort in research community has been paid on dealing with singularity analysis of robots.

Gracia studied the trajectory control algorithm for the conventional singular points and irregular singular points of the serial manipulator (Gracia L\&Andres J\&Tornero J, 2009), however, the singular configuration for 7DOF manipulator is much more complex, it is difficult to use this method to analyze redundant manipulator. Huang analyzed the singular situation of a 4DOF SCARA manipulator using moving frame method, and obtained the singular surface of the manipulator (Huang X\&Zhai M, 2014).

Bohigas proposed a numerical calculation method to get manipulator's singularity location( Bohigas O\&Zlatanov D\&Ros L\&Manubens M\&M.Porta,2014),this method is more appropriate for the calculation of low DOF manipulator, however ,the computation is greatly increased and the hardware requirements is relatively large with the increase of the freedom of manipulator.

Royer applied a geometric method to solve the singularity situation of a S-R-S configuration manipulator, and gives all the singular configurations(Royer L\&Bidard C\&Andriot C,1998), but it depends on observation ability to a large extends which is not intuitive and can't get the analytic expressions.

$\mathrm{Xu}$ studied the singularity and its avoidance algorithm of a non spherical wrist using a method based on Jacobean matrix elementary 
transformation, and gives all the singular configurations (Xu W\&Zhang J\&Liang B\&Li B, 2015), however, the method used in the article is not fully suitable for manipulator with spherical wrist. Similarly, Nokleby and Podhorodology successfully used the reciprocity-based methodology to identify the singularities of CSA/ISE STEAR test-bed manipulator a ground-based manipulator with link lengths and offsets, and Nokleby further analyzed the singularities of the SSRMS using the similar method $[8,9]$.

The method mentioned above all just solved the singularity situations for various manipulators, there is almost no analysis based on dexterity performance, and the kinematic characteristic of the manipulator near the singularity is not involved also.

The rest of the paper is organized as follows. Section 2 address the construction of kinematic model of 7DOF KUKA LBR iiwa 7 R800 robot. In section 3 , the Jacobean matrix of iiwa robot is analyzed based on screw theory, and the kinematic dexterity performance indices are derived based on the Jacobean matrix. In section 4 , the kinematic singularities of iiwa robot are derived based on the reciprocity product method and the singularity conditions and the singular direction are obtained. In section 5 , the numerical simulation is carried out based on dexterity performance index to validate the preceding analysis and Section 6 concludes the paper.

\section{Kinematic Analzsis of KUKA LBR Iiwa $7 \mathbf{R 8 0 0}$}

\subsection{Kinematic Model of KUKA LBR iiwa 7 $\mathbf{R 8 0 0}$}

KUKA LBR iiwa 7 R800 is a 7DOF universal lightweight redundant robot with large load and anthropomorphic characteristics. It can achieve good force feedback and force control, and avoid obstacles flexibly.

Moreover, it can achieve smooth control, the mechanical body of iiwa robot and its redundant features is depicted in figure 1.

Choosing the intersection point of joint 5,6 and 7 as the end coordinate system origin of the iiwa robot.

The entered link coordinate system for iiwa robot based on the Denavit-Hartenberg (DH) method is established in Fig.2, where $z_{i}$ is the rotation direction of the it $h$ joint.

The relevant parameters of iiwa robot are given in Table1.

Parameters $d_{1}, d_{3}$ and $d_{5}$ are the additional link offsets.

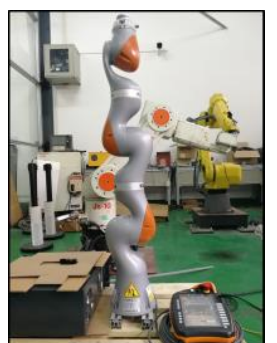

(a)

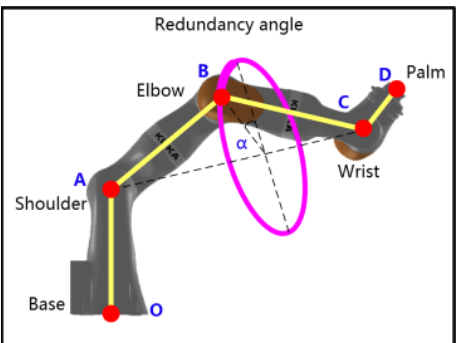

(b)
Figure 1: Mechanical structure and redundant angle of iiwa robot

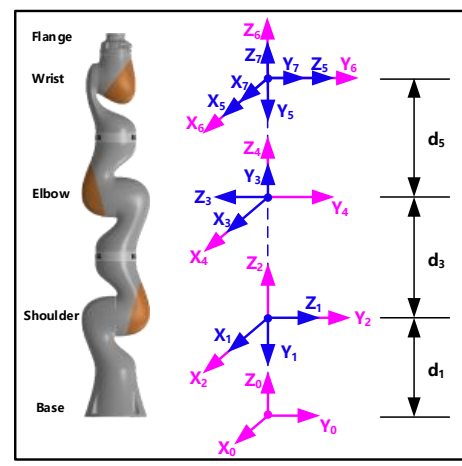

Figure 2: Link frame of iiwa robot

Table1. DH Parameter of Iiwa Robot

\begin{tabular}{|l|l|l|l|l|l|}
\hline \multirow{2}{*}{ Link } & \multicolumn{4}{|c|}{ DH parameters } & \multirow{2}{*}{ Range } \\
\cline { 2 - 5 } & $\theta_{i}$ & $\alpha_{i}$ & $a_{i}$ & $d_{i}$ & \\
\hline 1 & $\theta_{1}$ & -90 & 0 & 340 & $-170 \sim 170$ \\
\hline 2 & $\theta_{2}$ & 90 & 0 & 0 & $-120 \sim 120$ \\
\hline 3 & $\theta_{3}$ & 90 & 0 & 400 & $-170 \sim 170$ \\
\hline 4 & $\theta_{4}$ & -90 & 0 & 0 & $-120 \sim 120$ \\
\hline 5 & $\theta_{5}$ & -90 & 0 & 400 & $-170 \sim 170$ \\
\hline 6 & $\theta_{6}$ & 90 & 0 & 0 & $-120 \sim 120$ \\
\hline 7 & $\theta_{7}$ & 0 & 0 & 0 & $-175 \sim 175$ \\
\hline
\end{tabular}

\subsection{Forward Kinematics}

After determining the DH parameters of iiwa robot, only the joint Angle is the variable, and the forward kinematics equation of iiwa robot can be obtained through the general formula $T_{i}^{i-1}(q)$ of the homogeneous transformation matrix between the links:

${ }^{0} T_{7}(\theta)=\prod_{i=1}^{7}\left({ }^{i-1} T_{i}\left(\theta_{i}\right)\right)={ }^{0} T_{1}{ }^{1} T_{2}{ }^{2} T_{3}{ }^{3} T_{4}{ }^{4} T_{5}{ }^{5} T_{6}{ }^{6} T_{7}$

where $\theta_{i}$ is the joint variable. 


\section{Jacobian Matrix and Performance Indices}

The Jacobean matrix an indispensable matrix in understanding the motion of the end-effector, hence most kinematic performance measures are based on the Jacobean matrix and its evaluations.

Motion dexterity of Robot is one of the key research content of robot kinematics, and the manipulability, condition number and minimum singular value are the three classic index of dexterity evaluation of robot, in this paper, the manipulability and minimum singular value index are used to evaluate the dexterity of robot.

\subsection{Jacobian computation}

The Jacobean matrix of robot is defined as the generalized transmission ratio from joint space motion velocity to Cartesian space motion velocity:

$$
V=\left[\begin{array}{ll}
v & w
\end{array}\right]^{T}=\left[\begin{array}{ll}
J_{V} & J_{W}
\end{array}\right]^{T}=J(q) \dot{q}
$$

Where $w$ and $v$ represent the translational and rotation velocities of the end-effectors, $J(q)$ is the Jacobean matrix including the translational component $J_{V}$ and the rotation component $J_{W}$.

Traditional method used to solve the Jacobean matrix of robot mainly including vector product method, differential transform method and so on, however, the process of those method is very complex. In order to avoid a large number of formula calculation, this paper derives the Jacobean matrix of iiwa robot based on screw theory as proposed in (Kong X W\&Gosselin C M, 2005), and the Jacobean matrices will be uniformly referenced to a velocity point on the end effectors coincident with the origin of link frame5 and the orientation of the corresponding frame5, the screw coordinates for the ith joint in the Jacobean matrix defined with respect to frame 5 can be found:

$$
{ }^{5} \$_{i o_{5}}=\left[\begin{array}{c}
{ }^{5} \hat{z}_{i} \\
{ }^{5} \hat{z}_{i} \times{ }^{5} \hat{r}_{i \rightarrow o_{5}}
\end{array}\right]
$$

where ${ }^{5} \hat{z}_{i}$ is the unit vector giving the direction of joint $\mathrm{i},{ }^{5} \hat{r}_{i \rightarrow o_{4}}$ is the position vector from joint $\mathrm{i}$ to $O_{5}$.

Waldron et al demonstrated that the choice of an appropriate translational velocity reference point and reference orientation can greatly simplify the joint screw coordinate terms (Waldron K J\&Wang S L\&Bolin S J,1985).The selection of robot link coordinate system does not affect the analysis of the singular configuration of robot, therefore, a frame coincident with the coordinate system of joint5 is selected as reference frame to simplify expressions for the joints screws. For the chosen reference frame, the joint screws for the iiwa robot are:

$$
{ }^{5} \$_{1}=\left[\begin{array}{c}
c_{2} s_{4}-c_{3} c_{4} s_{2} \\
s_{2} s_{3} \\
c_{2} c_{4}+c_{3} s_{2} s_{4} \\
d_{3} c_{4} s_{2} s_{3} \\
d_{3} s_{4}\left(c_{2} c_{4}+c_{3} s_{2} s_{4}\right)-d_{3} c_{4}\left(c_{2} s_{4}-c_{3} c_{4} s_{2}\right) \\
-d_{3} s_{2} s_{3} s_{4}
\end{array}\right]
$$

${ }^{5} \$_{2}=\left[c_{4} s_{3}, c_{3},-s_{3} s_{4}, d_{3} c_{3} c_{4},-d_{3} s_{3},-d_{3} c_{3} s_{4}\right]^{T}$

${ }^{5} \$_{3}=\left[s_{4}, 0, c_{4}, 0,0,0\right]^{T}$

${ }^{5} \$_{4}=[0,-1,0,0,0,0]^{T}$

${ }^{5} \$_{5}=[0,0,1,0,0,0]^{T}$

${ }^{5} \$_{6}=\left[-s_{5}, c_{5}, 0,-d_{5} c_{5},-d_{5} s_{5}, 0\right]^{T}$

${ }^{5} \$_{7}=\left[c_{5} s_{6}, s_{5} s_{6}, c_{6},-d_{5} s_{5} s_{6}, d_{5} c_{5} s_{6}, 0\right]^{T}$

where $c_{i}$ and $s_{i}$ denote $\cos \theta_{i}$ and $\sin \theta_{i}$,respectively.

Then the Jacobean matrix for iiwa robot is:

$$
\begin{aligned}
{ }^{5} \$ & =\left[{ }^{5} \$_{1},{ }^{5} \$_{2},{ }^{5} \$_{3},{ }^{5} \$_{4},{ }^{5} \$_{5},{ }^{5} \$_{6},{ }^{5} \$_{7}\right] \\
& =\left[\begin{array}{ccccccc}
c_{2} s_{4}-c_{3} c_{4} s_{2} & c_{4} s_{3} & s_{4} & 0 & 0 & -s_{5} & c_{5} s_{6} \\
s_{2} s_{3} & c_{3} & 0 & -1 & 0 & c_{5} & s_{5} s_{6} \\
c_{2} c_{4}+c_{3} s_{2} s_{4} & -s_{3} s_{4} & c_{4} & 0 & 1 & 0 & c_{6} \\
d_{3} c_{4} s_{2} s_{3} & d_{3} c_{3} c_{4} & 0 & 0 & 0 & -d_{5} c_{5} & -d_{5} s_{5} s_{6} \\
J_{51} & -d_{3} s_{3} & 0 & 0 & 0 & -d_{5} s_{5} & d_{5} c_{5} s_{6} \\
-d_{3} s_{2} s_{3} s_{4} & -d_{3} c_{3} s_{4} & 0 & 0 & 0 & 0 & 0
\end{array}\right]
\end{aligned}
$$

where:

for overall evaluation of the manipulating ability of a $J_{51}=d_{3} s_{4}\left(c_{2} c_{4}+c_{3} s_{2} s_{4}\right)-d_{3} c_{4}\left(c_{2} s_{4}-c_{3} c_{4} s_{2}\right)$.

\subsection{Manipulability performance index}

Manipulability is measured as the product of the ellipsoid's axes, which reflects the ellipsoid's volume. Yoshikawa proposed the measure of manipulability robotic mechanism in positioning and orienting endeffectors (YOSHIKAWA T.1985).

Like most kinematic indices the manipulability index is based on the manipulator's Jacobean matrix. For a redundant manipulator the manipulability index is defined as the square root of the determinant of the product of the Jacobean matrix and its transpose: 


$$
\omega=\sqrt{\operatorname{det}\left(J(q) J^{T}(q)\right)}=\sqrt{\lambda_{1} \lambda_{2} \cdots \lambda_{m}}=\sigma_{1} \sigma_{2} \cdots \sigma_{m}
$$

where $J(q)$ is the velocity Jacobean matrix, $\lambda_{1}, \lambda_{2}, \cdots, \lambda_{m}$ are the eigenvalues of $J(q) J^{T}(q)$, and $\sigma_{1}, \sigma_{2}, \cdots, \sigma_{m}$ are the singular values of $J(q)$.

In order to compare mechanisms of different sizes and configurations fairly and reveal the transmission efficient from the joint space to the task space intuitively, Yashikawa further proposed the manipulability ellipsoid index on the basis of the definition of manipulability, as in Fig.3,it describes the dexterity of robot clearly and vividly on the geometrical level.

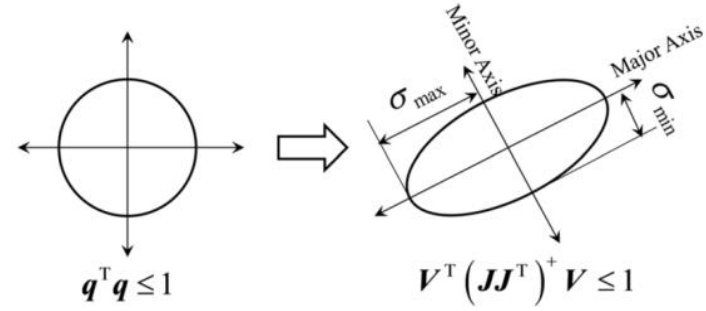

Figure 3: The manipulability ellipsoid

Therefore, the joint space is defined as a unit sphere:

$$
\|\dot{\theta}\|^{2}=\dot{\theta}_{1}^{2}+\dot{\theta}_{2}^{2}+\cdots+\dot{\theta}_{n}^{2} \leq 1
$$

It is mapped to an ellipsoid of the task space by the Jacobean matrix:

$$
V^{T}\left(J J^{T}\right)^{+} V \leq 1
$$

The direction of each axis of the ellipsoid is consistent with that of the eigenvector, and the length of each axis is equal to the reciprocal of the square root of its eigenvalue, which is also equal to the singular value. Obvious, when robot is in the singular configuration, the manipulability of the reference point of robot is zero, and the axis length of its corresponding manipulability ellipsoid in a certain direction also tends to zero, which degenerates into a plane.

\subsection{Minimun singular value performance index}

Minimum singular value index of Jacobean matrix represents the direction in which it is most difficult for the manipulator's end-effector to move, ignoring all other directions. It is the most effective measure of distance from singular configuration of robot (Klein C A, 1987).

According to the singular value decomposition theory of matrix, the singular value decomposition of Jacobean matrix is performed, as in:

$$
J(q)=U \Sigma V
$$

$$
\Sigma=\left[\begin{array}{ccccc}
\sigma_{1} & 0 & \cdots & 0 & 0 \\
0 & \sigma_{2} & \cdots & 0 & 0 \\
\vdots & \vdots & \ddots & \vdots & \vdots \\
0 & 0 & \cdots & \sigma_{m} & 0
\end{array}\right]
$$

Where $U \in R^{m \times n}, V \in R^{n \times n}$, and $\sigma_{1}, \sigma_{2}, \cdots, \sigma_{m}$ are the singular values of $J(q)$.

$$
\sigma_{\min }=\min \left(\sigma_{1}, \sigma_{2}, \cdots, \sigma_{m}\right)
$$

Obvious, when robot is near the singular configuration, the minimum singular value of the robot Jacobean matrix tends to zero, and the dexterity of robot gets worse.

\section{Kinematic Singularity on Screws Reciprocity}

The determinant of the Jacobean matrix of iiwa robot which is a 6x7 matrix cannot be derived directly, in this article, the method in $[14,15]$ is used to analyze the singularity of iiwa robot based on Jacobean matrix. The first step in applying the method of $[14,15]$ is to choose six linearly independent joint screws that denoted ${ }^{5} \$_{\text {sub }}$.Joint screws ${ }^{5} \$_{2}$ to ${ }^{5} \$_{7}$ from Eq.(11)were selected to form ${ }^{5} \$_{s u b}$ since the expressions for ${ }^{5} \$_{1}$ are the most complex:

$$
{ }^{5} \$_{\text {sub }}=\left[\begin{array}{cccccc}
c_{4} s_{3} & s_{4} & 0 & 0 & -s_{5} & c_{5} s_{6} \\
c_{3} & 0 & -1 & 0 & c_{5} & s_{5} s_{6} \\
-s_{3} s_{4} & c_{4} & 0 & 1 & 0 & c_{6} \\
d_{3} c_{3} c_{4} & 0 & 0 & 0 & -d_{5} c_{5} & -d_{5} s_{5} s_{6} \\
-d_{3} s_{3} & 0 & 0 & 0 & -d_{5} s_{5} & d_{5} c_{5} s_{6} \\
-d_{3} c_{3} s_{4} & 0 & 0 & 0 & 0 & 0
\end{array}\right]
$$

The determinant of ${ }^{5} \$_{s u b}$ is:

$$
\left|{ }^{5} \$_{s u b}\right|=d_{3} d_{5}{ }^{2} c_{3} s_{4}{ }^{2} s_{6}
$$

Therefore, there are three conditions where the matrix has a descending rank: (a) $c_{3}=0$, (b) $s_{4}=0$, (c) $s_{6}=0$. The singularity configuration of iiwa robot contains the above three conditions, additional singularity conditions can be obtained by means of the method in (Sugimoto K\&Duffy J\&Hunt K $\mathrm{H}, 1982$ ), that is, the product of a screw coordinate $W_{\text {recip }}$ which is orthogonal to the rest of the joint and the screw coordinate of joint 1 is solved. Each case is analyzed separately as follow: 


\section{$4.1 C_{3}=0$}

Set $c_{3}=0$ in Eq.(18).The matrix ${ }^{5} \$_{\text {sub }}{ }^{c_{3}=0}$ can be obtained as in:

$$
{ }^{5} \$_{s u b} \rightarrow c_{3}=0=\left[\begin{array}{cccccc}
c_{4} s_{3} & s_{4} & 0 & 0 & -s_{5} & c_{5} s_{6} \\
0 & 0 & -1 & 0 & c_{5} & s_{5} s_{6} \\
-s_{3} s_{4} & c_{4} & 0 & 1 & 0 & c_{6} \\
0 & 0 & 0 & 0 & -d_{5} c_{5} & -d_{5} s_{5} s_{6} \\
-d_{3} s_{3} & 0 & 0 & 0 & -d_{5} s_{5} & d_{5} c_{5} s_{6} \\
0 & 0 & 0 & 0 & 0 & 0
\end{array}\right]
$$

Let $\quad{ }^{5} W_{c_{3}=0} \otimes \$_{j}=0, \quad$ for $\quad j=2 \sim 7$, with $c_{3}=0$ allows the elements of ${ }^{5} W_{c_{3}=0}$ can be found as ${ }^{5} W_{c_{3}=0}=[0,0,1,0,0,0]^{T}$.

Note that ${ }^{5} W_{c_{3}=0}$ is not unique, there is an infinity of possible reciprocity screw quantities which are all scalar multiples of one another.

Setting the reciprocity product between ${ }^{5} W_{c_{3}=0}$ and ${ }^{5} \$_{1}$ to $\quad$ zero yields ${ }^{5} W_{c_{3}=0} \otimes{ }^{5} \$_{1}=-d_{3} s_{2} s_{3} s_{4}=0$, therefore, the additional singularity condition is obtained $s_{2}=0$.

Thus, if $c_{3}=0$ and $s_{2}=0$ then ${ }^{5} W_{c_{3}=0}$ is reciprocal to ${ }^{5} \$_{1}$ to ${ }^{5} \$_{7}$ the $c_{3}=0$ and $s_{2}=0$ defines a singularity configuration for iiwa robot.

\section{$4.2 \quad S_{4}=0$}

Set $s_{4}=0$ in Eq.(18). The matrix ${ }^{5} \$_{\text {sub }} \rightarrow_{4}=0$ can be obtained as in:

$$
{ }^{5} \$_{\text {sub }} \rightarrow s_{4}=0=\left[\begin{array}{cccccc}
c_{4} s_{3} & 0 & 0 & 0 & -s_{5} & c_{5} s_{6} \\
c_{3} & 0 & -1 & 0 & c_{5} & s_{5} s_{6} \\
0 & c_{4} & 0 & 1 & 0 & c_{6} \\
d_{3} c_{3} c_{4} & 0 & 0 & 0 & -d_{5} c_{5} & -d_{5} s_{5} s_{6} \\
-d_{3} s_{3} & 0 & 0 & 0 & -d_{5} s_{5} & d_{5} c_{5} s_{6} \\
0 & 0 & 0 & 0 & 0 & 0
\end{array}\right]
$$

Let ${ }^{5} W_{s_{4}=0} \otimes \$_{j}=0$, for $j=2-7$, with $s_{4}=0$ allows the elements of ${ }^{5} W_{s_{4}=0}$ can be found as ${ }^{5} W_{s_{4}=0}=[0,0,1,0,0,0]^{T}$. Setting the reciprocity product between ${ }^{5} W_{s_{4}=0}$ and ${ }^{5} \$_{1}$ to zero yields ${ }^{5} W_{s_{4}=0} \otimes{ }^{5} \$_{1}=0$.Thus, if $s_{4}=0$, then ${ }^{5} W_{s_{4}=0}$ is reciprocal to ${ }^{5} \$_{1}$ to ${ }^{5} \$_{7}$, the $s_{4}=0$ defines a singularity configuration for iiwa robot.

\section{$4.3 \mathrm{~S}_{6}=0$}

Set $s_{6}=0$ in Eq. (18). The matrix ${ }^{5} \$_{\text {sub }} \rightarrow_{6}=0$ can be obtained as in:

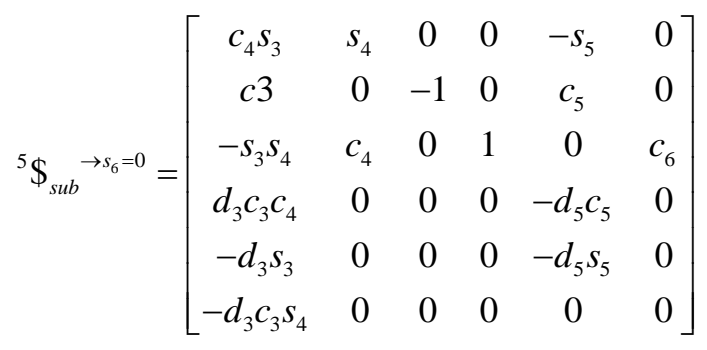

Let ${ }^{5} W_{s_{6}=0} \otimes \$_{j}=0$,for $j=2 \sim 7$, setting the reciprocity product between ${ }^{5} W_{s_{6}=0}$ and ${ }^{5} \$_{1}$ to zero with $s_{6}=0 \quad$ yields ${ }^{5} W_{s_{6}=0} \otimes{ }^{5} \$_{1}=0$, therefore, the additional singularity condition is obtained: (1) $s_{2}=0$;(2) $\quad c_{5}=0$.Thus, if $s_{6}=0 \& s_{2}=0$ or $s_{6}=0 \& c_{5}=0$, then ${ }^{5} W_{s_{6}=0}$ is reciprocal to ${ }^{5} \$_{1}$ to ${ }^{5} \$_{7}$, the $s_{6}=0 \& s_{2}=0$ and

$s_{6}=0 \& c_{5}=0$ defines the singularity configuration for iiwa robot.

Examining the singular configurations identified above, yields four unique set of conditions defining families of configurations resulting in singularity of iiwa robot are showed in Table2:

Table2. Singularity conditions for iiwa robot

\begin{tabular}{|l|l|}
\hline \multicolumn{1}{|c|}{ Number } & Singularity conditions \\
\hline 1 & $c_{3}=0 \& s_{2}=0$ \\
\hline 2 & $s_{4}=0$ \\
\hline 3 & $s_{6}=0 \& s_{2}=0$ \\
\hline 4 & $s_{6}=0 \& c_{5}=0$ \\
\hline
\end{tabular}


and the corresponding 3D configurations of iiwa robot is showed in Fig.4.

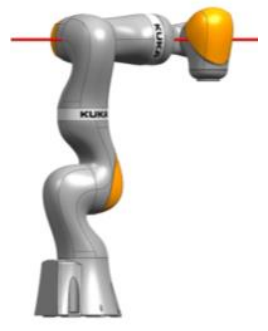

(1)

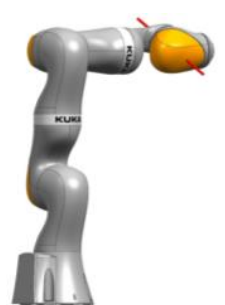

(3)

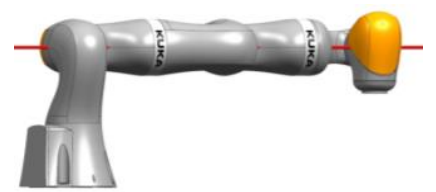

(2)

\section{Kinematic Singularity Simulation}

The singularity of iiwa robot which contains four kinds of singularity conditions is analyzed in the above analysis. In order to verify the above analysis, the singularity of iiwa robot is simulated and analyzed based on Robotics Toolbox as follow:

\section{$5.1 \quad c_{3}=0 \& s_{2}=0$}

Setting the other joint angle of iiwa robot except the joint 2 and joint 3 a constant. For joint2 and joint3, the range of motion is traversed to compute the manipulability and the minimum singular value of iiwa robot.

The relationship between joint 2 and joint 3 and manipulability of iiwa robot and minimum singular value of iiwa robot's Jacobean matrix is showed in Fig.5.

Figure 4: The singular configurations of iiwa robot
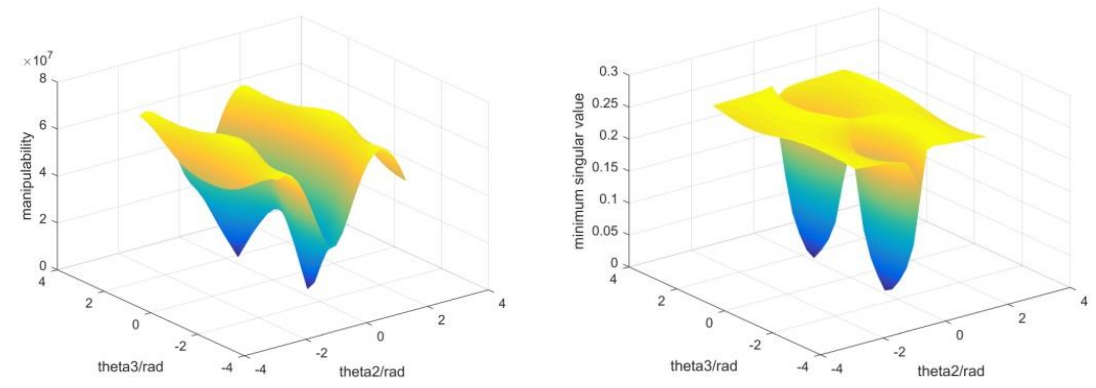

Figure 5: The manipulability and minimum singular value

\section{$5.2 \mathrm{~s}_{4}=0$}

Setting the other joint angle of iiwa robot except the joint 4 a constant.

For joint 4, the range of motion is traversed to

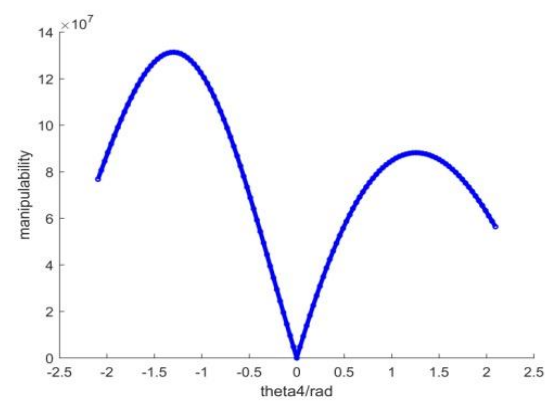

compute the manipulability and the minimum singular value of iiwa robot.

The relationship between joint 4 and manipulability of iiwa robot and minimum singular value of iiwa robot's Jacobean matrix is showed in Fig.6.

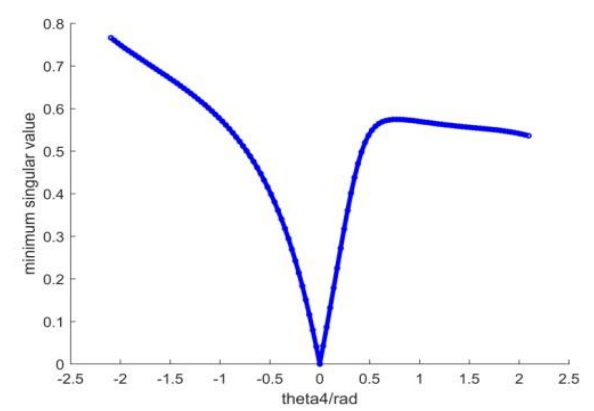

Figure 6: The manipulability and minimum singular value

\section{$5.3 \quad S_{6}=0 \& S_{2}=0$}

Setting the other joint angle of iiwa robot except the joint 6 and joint 2 a constant. For joint 6 and joint2, the range of motion is traversed to compute the manipulability and the minimum singular value of iiwa robot.

The relationship between joint 6 and joint 2 and manipulability of iiwa robot and minimum singular value of iiwa robot's Jacobean matrix is showed in Fig.7. 

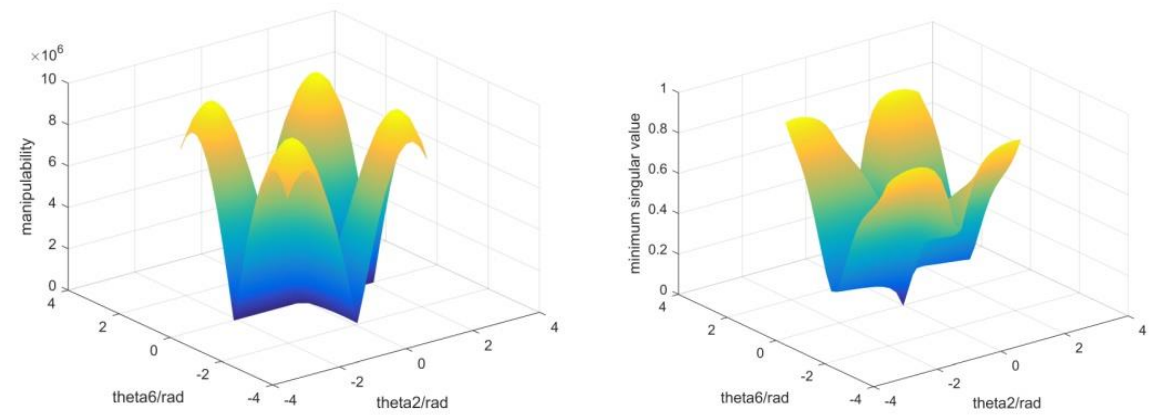

Figure 7: The manipulability and minimum singular value

\section{$5.4 \quad s_{6}=0 \& c_{5}=0$}

Setting the other joint angle of iiwa robot except the joint6 and joint5 a constant. For joint6 and joint5, the range of motion is traversed to compute the manipulability and the minimum singular value of iiwa robot the relationship between joint6 and joint5 and manipulability of iiwa robot and minimum singular value of iiwa robot's Jacobean matrix is showed in Fig. 8.
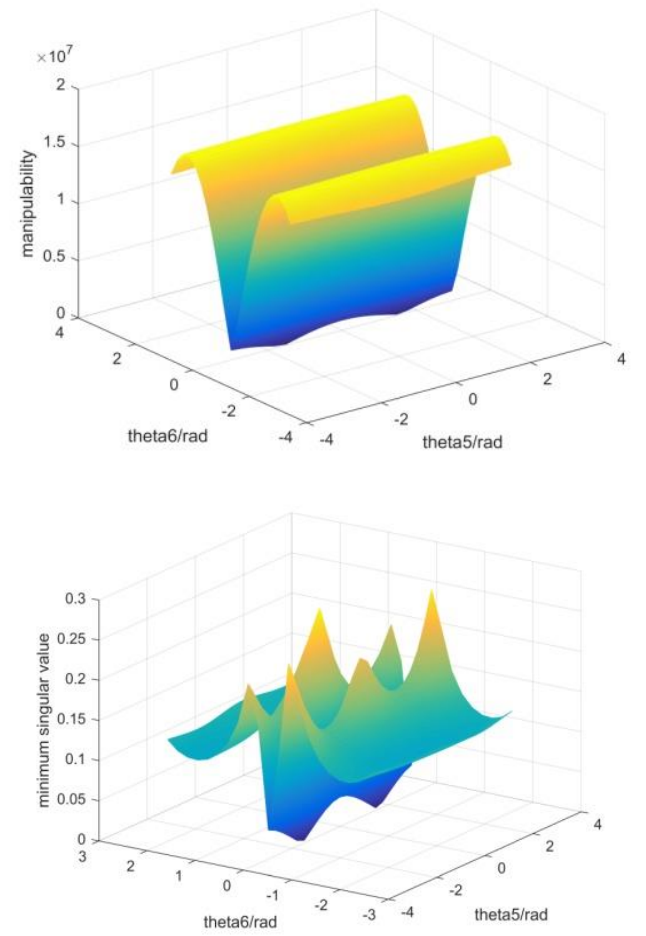

Figure 8: The manipulability and minimum singular value

When the iiwa robot is in one of the above singular position, a lack of DOF causes the robot to be unable to move in some certain direction.

The manipulability ellipsoid of the end-effector of iiwa robot is analyzed based on the above analysis, take the fourth example of the above singularity situation, the velocity manipulability ellipsoid of iiwa robot is shown in Fig. 9, the rest are similar.
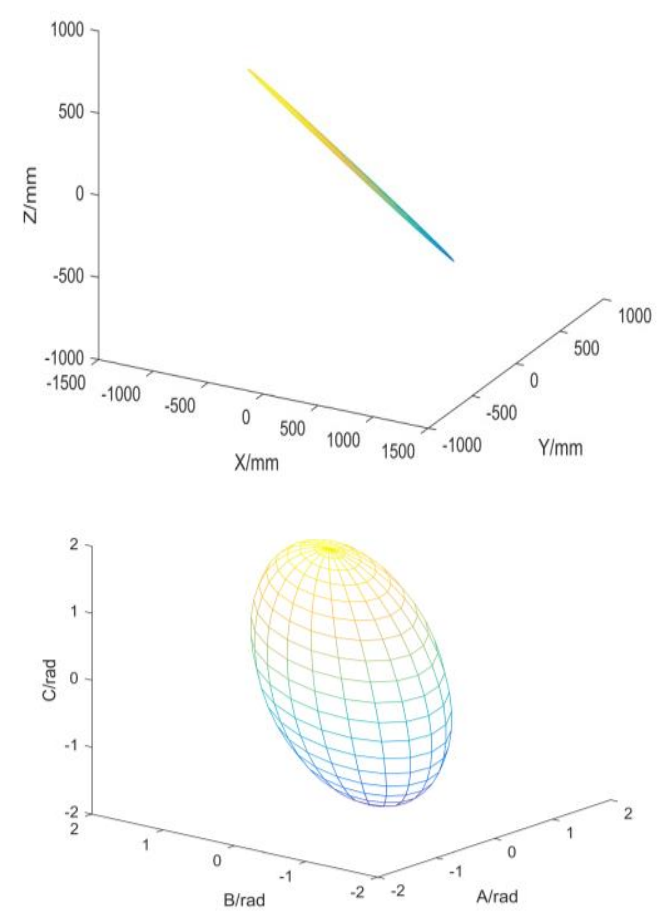

Figure 9: The translational and rotational velocity manipulability ellipsoidss

As can be seen from the above simulation results, there are four singularity conditions in all of iiwa robot. When the combination of the above four conditions occurs, the iiwa robot is also in the singular configuration. When iiwa robot is in the first singular configuration, that is $\theta_{2}=0 \& \theta_{3}= \pm \pi / 2$, the manipulability and the minimum singular value changed dramatically, both of them tends to zero as in Fig5,and the translational manipulability ellipsoid of the end point of the arm is degenerated into a plane, indicating that $\theta_{2}=0 \& \theta_{3}= \pm \pi / 2$ is a singular position of iiwa robot.

Similarly, $s_{4}=0, s_{6}=0 \& s_{2}=0$ and $s_{6}=0 \& c_{5}=0$ are all the singular configuration as in Fig. 6, Fig. 7, Fig. 8.

The simulation results further verify the correctness of the singularity analysis of the iiwa robot. 


\section{Conclusion}

Aimed at KUKA iiwa R800 robot which has seven DOF, firstly, the Jacobean matrix of iiwa robot is deduced based on the screw theory and then, the singularity problem of iiwa robot within its workspace was studied and resolved using the reciprocal product method in this paper, finally, the singularity of iiwa robot was simulated and analyzed using the Robotics Tool boxs based on the manipulability index and the minimum singular value index, and the analysis results further verifies the correctness of above theoretical analysis.

Theoretical analysis and simulation results show that there are four sets of conditions defining families of singular configurations resulting in a single-motion DOF loss for the iiwa robot. When iiwa robot is in a singular configuration, the manipulability and the minimum singular value of Jacobean matrix of the reference point at the end of iiwa robot tend to zero, and the manipulability ellipsoid of the end reference point of iiwa robot also degenerates into a plane with a lack of freedom and poor dexterity. Compared with traditional methods, the singularity analysis process is greatly simplified and the computation workload is greatly reduced and it would be the theory foundation to further research for subsequent singularity avoidance.

\section{Acknowledgment}

This work was supported by the Shanghai Municipal Science and Technology Commission (Grant No.15550721900 and 16DZ1120803)

\section{References}

[1] J. Gunn, R. Blackburn, and P. J. Taylor, Forensic Psychiatry: Clinical, Legal and Ethical Issues, Butterworth-Heinemann, Oxford, UK, 1993.

[2] M. W. Spong and M. Vidyasagar, Robot Dynamics and Control, JohnWiley \& Sons, 2008.

[3] Gracia L, Andres J, Tornero J. Trajectory tracking with a $6 \mathrm{R}$ serial industrial robot with ordinary and non-ordinary singularities[J]. International Journal of Control Automation \& Systems. 2009, 7(1): 85-96.

[4] Huang X, Zhai M. Singularity analysis of SCARA manipulator on moving frame method [J]. International Conforence on Mechanical Engineering and Mechanics. 2014.
[5] Bohigas O, Zlatanov D, Ros L, Manubens M, M.Porta.A General Method for the Numerical Computation of Manipulator Singularity Sets[J]. IEEE Transactions on Robotics. 2014, 30(2): 340-351.

[6] Royer L, Bidard C, Andriot C. Determination of Singularities and Self-Motion of a 7-dof Anthropomorphic Manipulator [M]. Springer Netherlands, 1998: 533-542.

[7] Xu W, Zhang J, Liang B, Li B. Singularity Analysis and Avoidance for Robot Manipulators With Nonspherical Wrists [J]. IEEE Transactions on Industrial Electronics. 2015, 63(1): 277-290.

[8] S. B. Nokleby, "On the Singular Configurations of the Canadarm2," in Proc. The 15th CISM/IFToMM Symposium on Theory and Practice of Robots and Manipulators, Montreal, Canada, pp. 1-10, 2004.

[9] Nokleby S B. Singularity analysis of the Canadarm2 [J]. Mechanism \& Machine Theory, 2007, 42(4):442-454.

[10] Kong X W, Gosselin C M. 'A dependent-screw suppression approach to the singularity analysis of a 7-DOF redundant manipulator: Canadarm2'[J]. Transactions- Canadian Society for Mechanical Engineering, 2005, 29(4):593604.

[11] Waldron K J, Wang S L, Bolin S J. A Study of the Jacobean Matrix of Serial Manipulators [J]. Journal of Mechanical Design, 1985, 107(2):230-238.

[12] YOSHIKAWA T. Manipulability of robotic mechanisms [J]. International Journal of Robotics Research, 1985, 4(2):3-9.

[13] Klein C A. Dexterity Measures for the Design and Control of Kinematically Redundant Manipulators [J]. Int.j. of Robotics Res, 1987, 6(2):72-83.

[14] S.B. Nokleby, R.P. Podhorodeski. Methods for resolving velocity degeneracies of jointredundant manipulators, in: J. Lenarčičn, M.M. Stanis`ic' (Eds.), Advances in robot kinematics, Kluwer Academic Publishers, London, United Kingdom, 2000, pp. 217-226.

[15] Nokleby S B, Podhorodeski R P. Velocity degeneracy determination for the kinematically redundant CSA/ISE STEAR testbed manipulator [J]. Journal of Field Robotics. 2000, 17(11): 633642.

[16] Sugimoto K, Duffy J, Hunt K H. Special configurations of spatial mechanisms and robot arms [J]. Mechanism \& Machine Theory, 1982, 17(2):119-13. 